\title{
The optimal gas dynamic design system of industrial centrifugal compressors based on Universal modeling method
}

\author{
Yuri Galerkin ${ }^{1}$, Aleksey Rekstin ${ }^{1}$, Aleksandr Drozdov ${ }^{1,{ }^{*}}$, Kristina Soldatova ${ }^{1}$, Olga Solovyeva ${ }^{1}$, and Elena Popova $^{1}$ \\ ${ }^{1}$ Peter the Great St.Petersburg Polytechnic University St.Petersburg, Polytechnicheskaya, 29, Russia
}

\begin{abstract}
We present the modern stage of development of Universal Modeling Method, a complex of mathematical models and software for optimal design of centrifugal compressors - a new version of simplified mathematical model of efficiency and new software for variation calculations of multistage compressors. Based on this numerical calculation complex we have created a method for preliminary design of flow paths of stages - 2D and 3D impellers, vane and vaneless diffusers and return channels. The new, 9th version of its mathematical model features a quasi-3D calculation method of $2 \mathrm{D}$ and $3 \mathrm{D}$ impellers design, a new principle of pressure characteristic calculation, a new model of vaneless diffusers and much more. "Digital twin of a centrifugal compressor stage" and "3D compressor" software create digital descriptions of the flow part and its solid model ("digital twin").
\end{abstract}

\section{Introduction}

Despite the successes of gas dynamic theory and computational gas dynamics, the issue of creating a reliable design of the centrifugal compressor flow path corresponding to the requirements of technical specifications for mass flow rate and pressure ratio at the highest possible efficiency has not been fully resolved.

Since an analytical description of the workflow is impossible, the search for the optimal solution can be done only by comparing the flow path options. For each of these options, it is necessary to calculate the efficiency and pressure ratio for a design flow. Russian and foreign methods of mathematical modeling [1 - 6] more or less correspond to the task.

The Polytechnic Scientific School of Compressor Engineering works with the Universal Modeling Method [7 - 9]. Using the 4th version of the Method, newgeneration gas pipe line compressors were created as part of the Ural-Gazprom conversion program [9] and dozens of other successful projects were implemented [10]. The mathematical models for calculating the efficiency are significantly improved in the models of the 5th version [11] and 6th version [12], and they are used in the design of new compressors $[13,14]$.

The disadvantage of the Method was the relative complexity of the design process. Design was carried out by consistent application of several indirectly related programs. During the process of optimization, the user needed to make a number of decisions requiring a lot of experience. Additionally, a solid model of a flow path was not created.

The aim of the research is to minimize the number of user interactions in the process of optimal design, improve the quality of mathematical models and create a closed system of computer programs. At the end of the design process, the developed end-to-end system produces a series of compressor gas-dynamic characteristics, and a solid model of the flow path. In order to improve the quality and reliability of the design, two main steps of design have been significantly improved - preliminary design and final design with the search for the optimal solution. The developed new design techniques and the improvement of mathematical models are based on computational and experimental researches.

\section{Variant calculation of a multistage compressor. New simplified mathematical model of efficiency}

Compliance with compressor parameters established by specification can be ensured with a virtually uncountable number of compressor variants. In general, designer can change: number of shafts on which the impellers are installed; number of stages on the shafts; shaft RPM; number of intermediate cooling; impellers diameters; types of impellers (2D or 3D); types of diffusers (vane or vaneless).

The same mass flow passes through all stages of the compressor, the volume flow is determined by the change in gas density $\rho_{0}^{*}$. One of the two main parameters of the design stage [0] - the design flow rate coefficient $\Phi_{\text {des }}$ depends on the impeller diameter $\mathrm{D}_{2}$ and the RPM $\mathrm{n}$. The second main design parameter is the design loading factor $\psi_{\text {Tdes }}$. The compressor pressure ratio $\pi^{*}$ is equal to the product of the pressure

* Corresponding author: A drozdi@mail.ru 
ratios of the stages (taking into account the pressure loss in the following gas cooler).

The idea of a mathematical model for evaluating the stages efficiency depending on the design parameters $\Phi_{\text {des }}, \psi_{\text {Tdes }}$, structural constraint $\overline{\mathrm{D}}_{\mathrm{h} \text { min }}$ and relative similarity criteria was formulated and implemented by $\mathrm{E}$. Popova [0]. The authors of [0] proposed and verified a modern version of the equations system:

$$
\eta_{\mathrm{des}}^{*}=1-\mathrm{X}_{1} \mathrm{~K}_{\Phi} \mathrm{K}_{\psi_{\mathrm{T}}} \mathrm{K}_{\mathrm{D}_{\mathrm{h}}} \mathrm{K}_{\mathrm{M}_{\mathrm{u}}}+\Delta \eta_{\mathrm{VD}}-\Delta \eta_{\text {inl }}
$$

where $\eta^{*}$ is efficiency, the coefficients $\mathrm{K}$ take into account the influence of the above parameters.

In expanded form, the equation system appears as follows:

$$
\begin{aligned}
& \eta_{\text {des }}^{*}= 1-\mathrm{X}_{1}\left[1+\mathrm{X}_{5}\left(\Phi_{\text {des }}-0.085\right)^{\mathrm{X}_{6}}\left(1+\mathrm{X}_{7} \overline{\mathrm{D}}_{\mathrm{h}}^{\mathrm{X}_{8}}\right)\right] \times \\
& \times\left[1+\mathrm{X}_{9}\left(\psi_{\text {Tdes }}-0.5\right)^{\mathrm{X}_{10}}\right] \times\left[1+\mathrm{X}_{11} \overline{\mathrm{D}}_{\mathrm{h}}^{\mathrm{X}_{12}}\left(1+\Phi_{\text {des }}\right)^{\mathrm{X}_{13}}\right] \times \\
& \times\left[1+\mathrm{X}_{14}\left(\mathrm{M}_{\mathrm{u}}-0.5\right)^{\mathrm{X}_{15}}(\Phi-0.01)^{\mathrm{X}_{16}}\right]+ \\
&+\mathrm{X}_{19}\left(\psi_{\text {Tdes }}-0.50\right)^{\mathrm{X}_{20}}-\mathrm{X}_{17} \frac{\Phi_{\text {des }}^{\mathrm{X}_{18}}}{\psi_{\text {Tdes }}}
\end{aligned}
$$

where $\bar{D}_{h}$ is relative hub ratio.

The availability of a method for assessing the efficiency of a stage opens up the possibility of calculating the efficiency of compressors taking into account losses in the inlet nozzle and intermediate coolers.

The free choice of RPM does not change the essence of the presented variant calculation, it only increases the number of compared variants.

For a compressor with a more complex scheme was created the PPMVTSK-G9R program, that provides an opportunity to compare variants with a different number of shafts, stages, loading factors and flow rate coefficients, the number and location of gas coolers. Examples of programs application for variant calculation are presented in [0].

After selecting the desired compressor variant, software handles the preliminary design - calculation of the compressor flow path sizes for calculating the gasdynamic characteristics by the Universal Modeling method.

\section{Preliminary impeller design}

In previous versions of the Method, the preliminary design of the stage flow path was carried out based on the principles of a Polytechnic scientific school [0] taking into account the recommendations of leading Russian scientists $[0-0]$. The experiments of the second half of the last century, on which design recommendations are based, do not reflect the complexity of modern compressor technology.

Since the Universal Modeling Method calculates the characteristics of any flow paths, automatically or interactively, the optimal design program is formally able to improve any preliminary project. However, the ability of a mathematical model to find the right solution should not be overestimated.

The most important part of the impeller design is an accurate estimate of the flow coefficient, at which the impeller blades, the diffuser vanes, and return channel ( $\mathrm{RCh}$ ) vanes have non-incidence inlet flow. It is also necessary to determine the loading factor with maximum accuracy.

The authors set the aim to solve these problems with the maximum approximation to reality already at the step of preliminary design, at which user participation is not necessary. This reduces the difference between the final project and the preliminary project.

To this end, $1242 \mathrm{D}$ and 3D impellers with design parameters in the range $\Phi_{\text {des }}=0.015-0.15, \psi_{\mathrm{Tdes}}=$ $0.40-0.70, \overline{\mathrm{D}}_{\mathrm{h}}=0.25-0.40$ were optimally designed by the Universal Modeling Method, and their blade rows were optimized based on the analysis of inviscid flow velocity diagrams. Both the main dimensions and the shape of the blades for each of the impellers are compared in many ways, so the proposed new preliminary design technique is based on the calculations of many hundreds of impeller variants.

The dimensions of the impeller entrance according to tradition, confirmed by successful practice, are selected on the basis of minimizing the relative velocity.

A well-tested recommendation - non-incidence flow around the blades at a set design mode - ended up ineffective in the case of low-flow impellers [0].

Comparison of dozens of variants of low-flow rate 2D impellers allowed to propose the following methodology for selecting sizes: inlet blade angle should be at least $23^{\circ}$; the inlet blade height corresponds to such a positive incidence angle, at which the peak of velocity at the leading edge is dosed - the outlet blade angle should be at least $16^{\circ}$, etc.

The preliminary design of medium-flow rate $2 \mathrm{D}$ impellers and high-flow rate $3 \mathrm{D}$ impellers basically corresponds to that presented in the monograph by $\mathrm{Yu}$. B. Galerkin [0]. The results of researches by CFD methods from $[0,0]$ were used. The main novelty of the preliminary design is the exact recommendations on choosing the sizes of the flow path needed to calculate the characteristics according to the efficiency model of the Universal Modeling Method.

For sizes $\bar{D}_{1 s}$ (blade inlet shroud diameter), $\bar{D}_{0}$, $\bar{D}_{h}, \bar{L}_{m}$ (relative axial length of impeller), $\bar{D}_{1 h}$ (blade inlet hub diameter), $\bar{R}_{h}$ (relative hub radius of curvature), $\bar{R}_{s}$ (relative shroud radius of curvature), $l_{m b l}$ (length of a blade in meridional plane), $\bar{b}_{2}$ (relative outlet blade height), formulas that approximate the results of a computational research are recommended for calculating the inlet blade angles at the shroud and at the hub $\beta_{\mathrm{b} 11 \mathrm{~s}}{ }^{0}, \beta_{\mathrm{bl1h}}{ }^{0}$, the outlet blade angle $\beta_{\mathrm{b} 12}{ }^{0}$, the number of blades $z_{\text {imp }}$ and the shape factors of the blades in the 3DM.023 program. 


\section{Preliminary design of stator stage elements}

Stator elements of centrifugal compressor stages consist of vane and vaneless diffusers, return channels.

Modern vaneless diffusers have a narrowed initial section to increase the flow angle to the main part with a constant width. If the flow angle at the exit of the impeller is sufficient for the VLD to work well, a narrowed initial section is not needed. Preliminary design subjects are relative width $\bar{b}_{3}=b_{3} / D_{2}$ (where $b_{3}$ is VLD width) and radial extent $\bar{D}_{4}=D_{4} / D_{2}$ (where $\mathrm{D}_{4}$ is VLD outlet diameter). In the proposed method of preliminary design, the choice of the relative width is linked for the first time not with the parameters of the design mode, but with ensuring a non separate flow at the pre-surge mode.

\subsection{Assessment of the surge limit}

In the practice of calculations, it is generally accepted to understand the surge line as a regime with a maximum pressure ratio [0].

The surge line corresponds to a flow coefficient at which the head polytropic coefficient $h_{p}=h_{T}-h_{w}$ (where $h_{p}$ is polytropic head; $h_{T}$ is Euler head; $h_{w}$ is loss of head) reaches its maximum:

$$
\frac{d\left(\psi_{p}\right)}{d \Phi}=0
$$

The linear dependence of the loading factor on the relative flow coefficient was analyzed in publications $[0$, $0]$. Within the practically used part of the compressors dimensional characteristics, the dependence of the work coefficient on the flow rate is also linear [0]. For preliminary design purposes, it is acceptable to consider the function $\psi_{T}=f(\Phi)$ to be linear as well, although the dependence between $\Phi$ and $\varphi_{2}$ is not quite linear (where $\varphi_{2}$ is flow rate coefficient at an impeller exit). After transformations and a search for $\Phi_{c r}$ corresponding to the maximum value of $\psi_{p \max }$ :

$$
\frac{\Phi_{c r}}{\Phi_{d e s}}=1-\left[\frac{\frac{\psi_{T 0}}{\psi_{\text {des }}}-1}{17\left(1-\eta_{\text {hyd des }}\right)}\right]^{0,5}
$$

\subsection{Choosing the width of the VLD main part}

After calculating the ratio of $\Phi_{c r} / \Phi_{\text {des }}$, it is necessary to determine how much the exit flow angle from the impeller $\alpha_{2 \text { des }}$ (where $\alpha_{2 \text { des }}$ is the flow angle at design flow rate) decreases at the surge line. The obtained value should be compared with the angle $\alpha_{3 s b}$ (where $\alpha_{3 s b}$ is the flow angle at VLD inlet) at which separation occurs in the main part of the VLD ("separation boundary"), and choose $\bar{b}_{3}$ at which $\alpha_{3 c r}=\alpha_{3 s b}$.

The strong dependence of the critical flow angle at which separation begins on the relative width of the VLD is obvious. Processing the results of the calculation experiment established the following dependence of the tangent of the flow angle at the separation boundary in the VLD, i.e. on the surge line:

$$
\operatorname{tg} \alpha_{3 s b}=0.0875+3.5 \bar{b}_{3}
$$

The relative width of the VLD at which $\alpha_{2 c r}=\alpha_{2 s b}$ :

$$
\bar{b}_{3}=\frac{-0.0875+\sqrt{0.00766+14 \operatorname{tg} \alpha_{2 s b} \bar{b}_{2}}}{7}
$$

\subsection{VLD radial extent}

Even with an inlet flow angle $10^{\circ}$, in a narrow and long diffuser static pressure increases $[0,0]$. Obviously, a rational ratio $D_{4} / D_{2}$ should be sought in conjunction with the subsequent element of the flow path. In [0] the problem is discussed on the basis of the expression for the loss of efficiency in the stator elements of the stage. Below this expression is transformed like this:

$$
\zeta_{\mathrm{SE}}=\zeta_{\mathrm{VLD}}+\frac{\zeta_{\mathrm{RCh}}}{\overline{\mathrm{D}}_{4}^{2}}\left[\operatorname{ctg}^{2} \alpha_{2}+\left(\frac{\operatorname{tg} \alpha_{2}}{\mathrm{~b}_{3} / \mathrm{b}_{2}}\right)^{2}\right]=\mathrm{f}\left(\overline{\mathrm{D}}_{4}\right) \rightarrow \min (7)
$$

where $\zeta$ is the loss coefficient.

With an increase in the radial extent of VLD, the loss coefficient increases, but the kinetic energy at the RCh inlet or scroll becomes smaller. In a longer RCh friction losses are higher. Parameters analysis of the series of model stages $21 \mathrm{CV}$ developed by K.V. Soldatova [0] and the calculation research by the Universal Modeling Method made it possible to propose the following equation for the preliminary design of VLD in stages with RCh:

$$
\bar{D}_{4}=1.45+1.4\left(\Phi_{\text {des }}-0.015\right)^{0.5}
$$

For stages with scrolls, the radial extent of the VLD is limited by design considerations.

\subsection{Preliminary RCh design}

In the formation of the design method, the results, generalized in the monograph [0] and a number of additional calculations are used.

Characteristic features of RCh vanes by the Universal Modeling Method: the shape of the vane midline is a circle arc; the shape of the vane profiles is a two-arc profile with a maximum thickness in the middle of the profile equal to $\delta_{\max } / D_{2}=0.040$; outlet vane angle $\alpha_{v 6}=95^{\circ}$; the number of vanes is even; the number of straightening vanes is half the number of main vanes. 
In [0] the characteristics of the efficiency and work coefficient of one of the 20CE stages series compares, obtained as a result of repeated tests of different stage versions. On average, the " 24 vanes with double-arc profile" vane row increases the stage efficiency by $1.2 \%$.

According to the experience of model stages series designing [0, 0] and CFD calculations [0], RCh vanes with an inlet vane angle of less than $25^{\circ}$ are ineffective. Empirical formulas are recommended for the range of stages $\Phi_{\text {des }}=0.015-0.15$ :

$$
\begin{gathered}
\alpha_{v 5}^{\mathrm{o}}=25^{\mathrm{o}}+21.6^{\circ}\left(\Phi_{d e s}-0.015\right)^{0.35} \\
\frac{\bar{b}_{5}}{\bar{b}_{4}}=1.45-0.5\left(\Phi_{\text {des }}-0.015\right)^{0.4} \\
z_{R C h}=20+12\left(\Phi_{\text {des }}-0.015\right)^{0.35}
\end{gathered}
$$

where $\bar{b}_{5}$ is vane height at RCh inlet; $z_{R C h}$ is number of $\mathrm{RCh}$ vanes; $\alpha_{v 5}^{\circ}$ is vane angle (to tangential direction) at $\mathrm{RCh}$ inlet.

The vanes height at the outlet is determined from the condition of mandatory acceleration of the flow in the outlet annular confuser (OAC) of at least $15 \%$ and the need for a smooth rotation of the flow in the OAC [0].

Given the accepted ratios $R_{s 6} / b_{6}=0.6$ and $F_{6} / F_{0^{\prime}}=$ 1.15 (where $\bar{b}_{6}$ is blade height at RCh outlet):

$$
\bar{b}_{6}=0.417\left[\left(\bar{D}_{0^{\prime}}^{2}+1.38\left(\bar{D}_{0^{\prime}}^{2}-\bar{D}_{h^{\prime}}^{2}\right)\right)^{0.5}-\bar{D}_{0^{\prime}}\right]
$$

The $\mathrm{RCh}$ vane outlet diameter is equal to:

$$
\bar{D}_{6}=\bar{D}_{0^{\prime}}+2 \bar{R}_{s 6}=\bar{D}_{0^{\prime}}+2 \frac{R_{s 6}}{b_{6}} \bar{b}_{6}=\bar{D}_{0^{\prime}}+1.2 \bar{b}_{6}
$$

where $\bar{D}_{6}$ is RCh outlet diameter.

\subsection{Capabilities of the preliminary design method}

An example of a quick analysis of the stages efficiency with different design parameters is shown in the data below for calculating stages with $\Phi_{\text {des }}=0.015,0.025$, $0.045,0.0625$ and 0.080 . All stages have identical remaining design parameters $\psi_{\text {Tdes }}=0.050, \quad \overline{\mathrm{D}}_{\mathrm{h}}=$ 0.350 , relative Mach number $M_{u}=0.70$, impeller diameter Reynolds number $\operatorname{Re}_{\mathrm{u}}=6000000$, isentropic coefficient $\mathrm{k}=1.40$. Figure 1 (left) shows the characteristics of efficiency, polytropic work coefficient, work coefficient and pressure ratios of a stages series.

After optimizing the flow path sizes during the final design, the efficiency can be increased. But for a comparative assessment, the data presented are sufficient.
The effect of $\Phi_{\text {des }}$ on the stage characteristics is logical. Efficiency reaches its maximum at such $\Phi_{\text {des }}$ that the decrease in friction loss with increasing channels hydraulic diameter ceases to compensate for the growth of the lost pressure from an increase in the growth of flow velocities in the impeller and in stator elements (SE).

\section{The main features of the Universal Modeling Method 9th version}

In the end-to-end system, after the preliminary design, optimization of the flow path main dimensions and calculation of the gas-dynamic characteristics of the compressor are carried out by the 9th version of the model. To calculate the compressor characteristics, mathematical models of efficiency and work coefficient are used. In the 9th version, both models are different from the previous ones. An example of a formula for calculating the static efficiency of the impeller demonstrates the general features of the models of the Universal Modeling Method and the features of the 9th efficiency model:

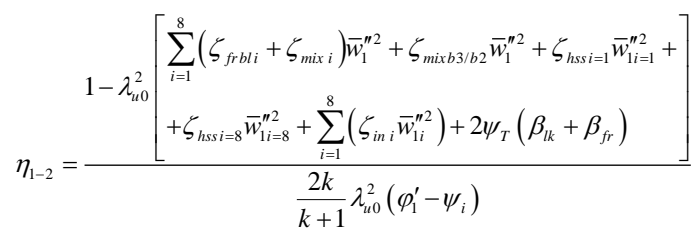

where $\lambda$ is velocity coefficient; $\bar{w}_{1}^{\prime \prime}$ is relative velocity in impeller throat; $\varphi_{1}^{\prime}$ is flow rate coefficient at an impeller inlet.

The structure of the formula follows from the wellknown connection of efficiency with the indicators of polytropic and isentropic. As in all models of the Method, friction, mixing, and incidence losses are calculated separately. Losses are calculated on the blades (separately on both sides) and on the hub and shroud surfaces. Loss coefficients are algebraic equations with empirical coefficients. A feature of the 9th model for the impeller is that profile losses are calculated on eight axisymmetric current surfaces. This brings the impeller geometric model to the real flow path.

In papers $[0,0]$ it is shown the linear characteristic $\psi_{\mathrm{T}}=\mathrm{f}\left(\varphi_{2}\right)$, on which the loading factor model in 9th version is based. Two parameters determine the nature of the loading factor characteristic:

$$
\psi_{\mathrm{T}}=\psi_{\mathrm{T} 0}-\varphi_{2} \cos \beta_{\mathrm{T}}
$$

where:

$$
\begin{aligned}
& \beta_{T}^{0}=10.9655\left(\beta_{b l 2}^{0}\right)^{2}+17.4802\left(\frac{l}{t}\right)^{0.4}+ \\
& +18.22 \bar{b}_{2}^{0.5}-5.702\left(\frac{b_{2}}{b_{1}}\right)^{0.8}
\end{aligned}
$$



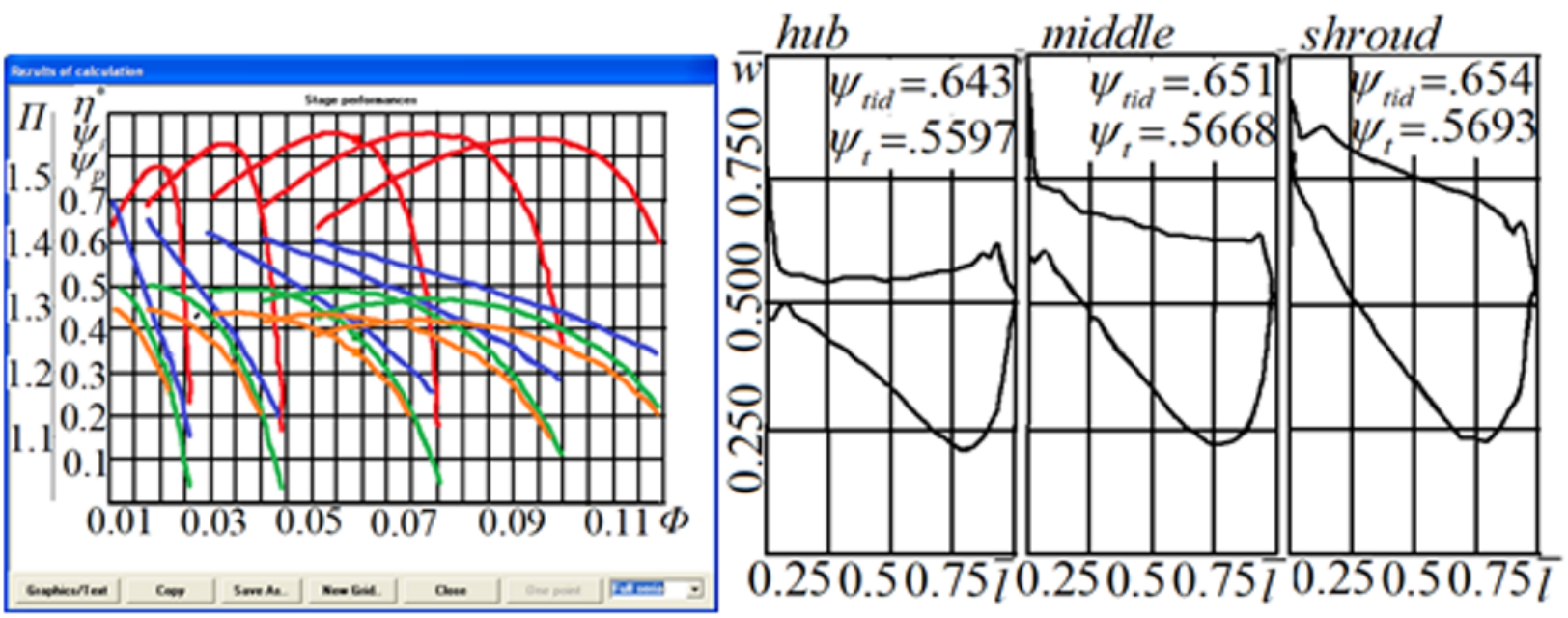

Fig. 1. “OPTIM 2" program, preliminary design. A series of stages with $\Phi_{\text {des }}=0.015,0.025,0.045,0.0625$ and 0.080 . Red efficiency, blue - work coefficient, red - polytropic work coefficient, green - pressure ratio (left); 3DM.023 program. The final project of the 3D impeller. Velocity diagrams on three streamlines (right).

$$
\begin{aligned}
& \psi_{T 0}=1-0.0479\left(\frac{l}{t}\right)^{0.9}-0.0025\left(\frac{b_{2}}{b_{1}}\right)^{2}+ \\
& +0.0255\left(\frac{\beta_{b l 2}}{40}\right)^{0.7}-3.7462 \bar{b}_{2}^{1.5}
\end{aligned}
$$

Unlike the well-proven but complex mathematical model of VLD of previous versions, the introduced model is based on a generalization of the calculated CFD experiment $[0,0,0]$. To calculate the parameters at the VLD outlet, it is sufficient to know the loss coefficient and the flow angle at the VLD outlet. In [Ошибка! Источник ссылки не найден.] approximating equations of the form $\zeta, \alpha_{4}=f\left(\bar{b}_{2}, \bar{D}_{4}, \bar{\kappa}_{r g}, \alpha_{2}, \lambda_{c 2}, \operatorname{Re}_{b 2}\right)$ were proposed, where $\bar{\kappa}_{r g}$ is relative surface roughness.

\section{Optimization of the flow path and calculation of characteristics}

After the preliminary design, the files necessary for calculating the velocity diagrams of the inviscid quasithree-dimensional flow on the impeller blades are formed.

Advantages of the preliminary design of the blade row:

- the loading factor corresponds to its design value;

- the general nature of the flow around blades satisfies the requirements formulated in [0].

The disadvantage of the preliminary design is that the cross-section at the row inlet is slightly smaller than necessary, therefore, at the shroud streamline there is a peak of velocities corresponding to a negative incidence angle.

In the final project, to eliminate the negative incidence angle, it turned out to be sufficient to slightly increase the impeller entrance diameter $\overline{\mathrm{D}}_{0}$. According to [0] it is advisable to equalize the circulation of velocity on different axisymmetric current surfaces.

This is due to a variable outlet angle $\beta_{\mathrm{bl} 2}$ with an increase from the hub to shroud. To reduce the unevenness of the meridional flow, the blades are slightly inclined in the direction opposite to the rotation direction. Figure 1 (right) shows velocity diagrams on three axisymmetric streamline surfaces of the final impeller version.

Characteristics calculated by the program "OPTIM 2 " showed that the correction of the stator elements basic dimensions is not required. 3D impeller size changing increased the efficiency at the design point by only $0.2 \%$. More importantly, the adjustment of the impeller dimensions provides non-incidence flow around the 3D impeller blades at the shroud streamline.

\section{Example of optimal compressor design}

The design of a single-stage compressor for a turboexpander unit was made for one of industrial partners. Figure 2 shows a compressor cross-section and results of preliminary design, efficiency and other parameters of candidates with different loading factors.

The hub ratio is big that influences efficiency. The compressor candidate with $\psi_{T \text { des }}=0.435$ was chosen.

The candidate has slightly lesser efficiency than candidates with larger loading factors. But the chosen candidate has a wider operating area towards lower flow rates, and the maximum power coincides practically with the design flow rate. This is important for a turbine drive.

For the selected candidate, the PC program automatically performs the preliminary design. By preliminary design the radial impellers have blades with controlled velocity diagrams. For a cast impeller of a turbo-expander compressor, a simpler midline is adopted - a circular arc. At the final design, the vanes of the 

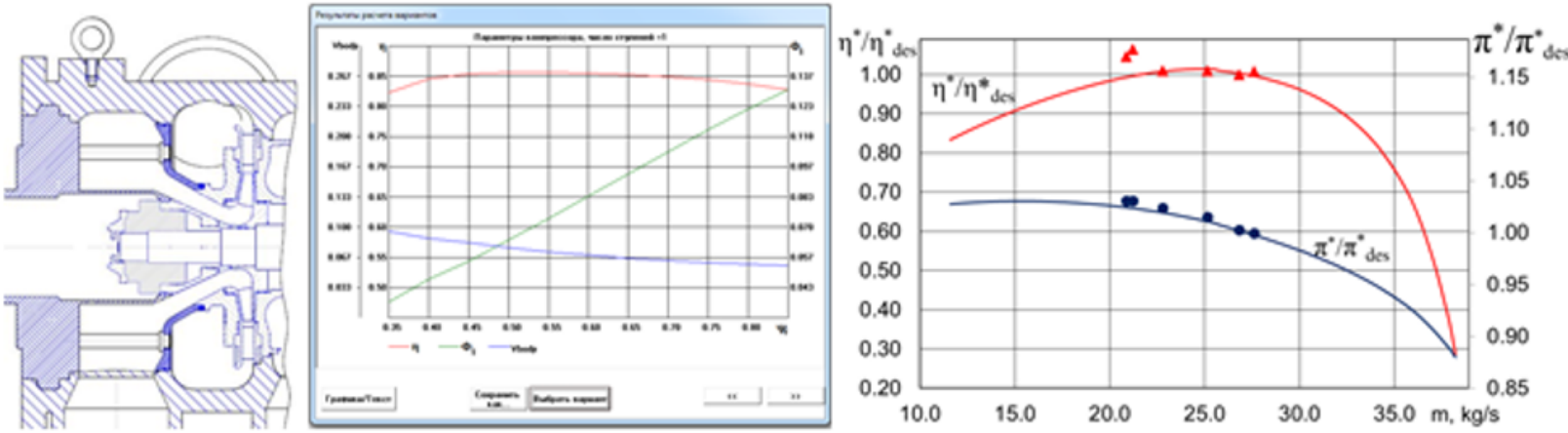

Fig. 2. Turbo-expander compressor, preliminary design program menu and compressor candidates with different loading factor $\psi_{\mathrm{T} \text { des }}=$ var (above), design characteristics (solid line) and the results of plant test (dots).

diffuser were adjusted for a set design flow rate of 27.26 $\mathrm{kg} / \mathrm{s}$. The design characteristics of the compressor are shown in Figure 2.

The factory "air" compressor tests are recalculated to the design conditions shown by dots in Figure 2. The tests confirmed the design level of efficiency and showed a margin in pressure ratio. Cast impellers have irregular shapes. The margin guarantees necessary pressure ratio for impellers those shape deviates of design one.

\section{Conclusion}

The authors constantly use the engineering methodology in design work and are engaged in the improvement of this tool. In the near future, it is planned to carry out an extensive analysis of vane diffusers and return channels by CFD calculations, approximate the results and use simpler and more reliable math models of these elements in the compressor stage model. Another task is the accumulation of experimental data on the characteristics of high flow rate stages with 3D impellers.

\section{Acknowledgements}

The research was performed by a Grant of the President of the Russian Federation for young PhD MK-1893.2020.8.

\section{References}

[1] A.T. Lunev, Compressors and pneumatics 10, 4-7 (2001)

[2] A. Lunev, O.A. Viachkilev, Y.V. Drozdov, Design and research of compressor machines $\mathbf{3}$ (1997)

[3] A.T. Lunev, Development of high-efficiency replacement flow parts of centrifugal compressors of gas-pumping units: Dis ... cand. tech. sciences (2005)

[4] D. Japikse, Design system development for turbomachinery (turbopump) designs and a decade beyond, JANNAF Conference (1998)

[5] D. Japikse, Agile design system in the age of concurrent engineering, JANNAF Conference (1996).
[6] D. Japikse, J. Bitter, Effective two-zone modeling of diffusers and return channel systems for radial and mixed-flow pumps and compressors, 11th International symposium on transport phenomena and dynamics of rotating machinery (2006)

[7] Y. Galerkin. Turbocompressors (Information and publishing center KHT, 596, 2010)

[8] Y. Galerkin, Proceedings of the science school of compressor engineering SPbGPU (M.: pub. SPbGPU, 2000)

[9] Y. Galerkin, Proceedings of the science school of compressor engineering SPbGPU (M.: pub. SPbGPU, 2010)

[10] Yu. Galerkin, A. Rekstin, K. Soldatova, A. Drozdov, Yu. Popov, The development of the scientific school of turbocompressor engineering LPI-SPbPU Peter the Great, the results of cooperation with compressor builders, Proceedings of 17th International Scientific and Technical Conference, Kazan (2017)

[11] K.V. Soldatova, Creation of a new mathematical model of the flowing part of centrifugal compressors and a database of model stages: Dis ... doc. tech. sciences (2015)

[12] A. Drozdov, Design method of centrifugal compressors with 3D impellers: Cand. of tech. Sci. (2017)

[13] A.F. Rekstin, V.B. Semenovskiy, K.V. Soldatova, Y.B. Galerkin, K.K. Sokolov, Compressors and pneumatics 1, 13-20 (2018)

[14] A.F. Rekstin, B.V. Bakaev, St. Petersburg Polytechnic University Journal of Engineering Science and Technology 4, 24-38. DOI: 10.18721/JEST.24403 (2018)

[15] K. Seleznev, Y. Galerkin, Centrifugal compressors (1982)

[16] E. Popova, Optimization of the main parameters of turbomachine stages on the basis of mathematical modeling: Dis ... cand. tech. sciences (1990)

[17] A.F. Rekstin, E.Y. Popova, A.A. Uchekhovsky, Compressors and pneumatics 1, 33-40 (2018)

[18] V.F. Ris, Centrifugal compressors (1964)

[19] G.N. Den, The mechanics of the flow in centrifugal compressors (1973)

[20] V. Schnepp, Design and calculation of centrifugal compressor machines (1995) 
[21] C.P. Livshits, Aerodynamics of centrifugal compressor machines (1966)

[22] A.F. Rekstin, Y.B. Galerkin, Bulletin of the Perm national research Polytechnic University 2, 4354. DOI: 10.15593/2224-9877/2018.2.06 (2018)

[23] Y.B. Galerkin, A.F. Rekstin, K.V. Soldatova, A.A. Drozdov, Compressors and pneumatics 4, 21-31 (2015)

[24] J.M. Sorokes, Sidestream optimization through the use of computational fluid dynamics and model testing turbomachinery sympos, 2000.

[25] Yu.B. Galerkin, K.V. Soldatova, Compressors and pneumatics 1, 24-34 (2016)

[26] Yu. Galerkin, A. Rekstin, K. Soldatova, A. Drozdov, Compressors and pneumatics 6, 11-19 (2016)

[27] Yu. Galerkin, O. Solovieva, Compressor technology and pneumatics 3, 35-41 (2014)

[28] Yu. Galerkin, O. Solovieva, Compressor technology and pneumatics 4, 15-21 (2014)

[29] A.F. Rekstin, K.V. Soldatova, Y.B. Galerkin, Proceedings of higher educational institutions 9, 702/2018. DOI: $10.18698 / 0536-1044-2018-9-44-$ $56(2018)$

[30] L. Marenina, Compressor technology and pneumatics 3, 27-35 (2016)

[31] A.F. Rekstin, A.A. Drozdov, O.A. Solovyeva, Y.B. Galerkin, AIP Conf. Proc. 2007, 030035-1030035-12. DOI: $10.1063 / 1.5051896$ (2018)

[32] O.A. Solovyova, A mathematical model for calculating the gas-dynamic characteristics and optimizing the vaneless diffusers of centrifugal compressor stages: PhD thesis (SPbPU, 2018) 